\title{
La Dette gidienne de Marguerite Yourcenar
}

\author{
David Keypour \\ (University of Western Ontario)
}

Un rapprochement entre Alexis ${ }^{1}$ de Marguerite Yourcenar écrit en 1929 et l'oeuvre de Gide s'impose à plusieurs titres. Tout d'abord pour des raisons d'histoire littéraire que la romancière elle-même ne manque pas de signaler. Mais aussi pour des raisons de lecture intertextuelle; et si l'on s'intéresse au féminisme en littérature, pour ce que, en regard de l'oeuvre de Gide, la problématique de l'homosexualité masculine est traitée ici par une femme.

Dès sa couverture, le livre de Yourcenar invite à faire ce rapprochement. Comme elle le rappelle elle-même dans sa préface de 1963, le titre de son livre est emprunté à la deuxième Eglogue de Virgile. Gide avait fait de même pour le titre de son Corydon. Ici le cousinage des deux oeuvres s'affirme par référence à un même auteur classique et à une oeuvre qui, sur le mode lyrique, touche au même thème commun.

Le sous-titre de Yourcenar est une allusion directe à un des premiers écrits gidiens. Elle appelle son Alexis également Le Traité du vain combat, de même que Gide avait sous-titré sa Tentative amoureuse, Le Traité du vain désir. Cette fois, la référence est textuelle à un mot près.

Mais, "en dépit de ce rappel, insiste Yourcenar dans sa préface, l'influence de Gide fut faible sur Alexis." Et plus loin:

"Des grands livres de Gide où le sujet qui m'occupe était enfin ouvertement traité, la plupart ne m'étaient encore connus que par ouï-dire; leur effet sur Alexis tient bien moins à leur contenu qu'au bruit fait autour d'eux, à cette espèce de discussion publique s'organisant autour d'un problème jusquelà examiné en huis-clos, et qui m'a certainement rendu plus facile d'aborder sans trop d'hésitation le même thème."(p. 17)

LittéRéalité, Vol. I, No. 1, Printemps/Spring 1989 
On serait amené à faire beaucoup de réserve face à cette dénégation quand on pénètre dans le détail du texte d'Alexis. Mais examinons seulement la dette que l'auteur veut bien reconnaître à l'égard de Gide:
C'est du point de vue FORMEL surtout [écrit-elle en italique] que la lecture des premiers livres de Gide m'avait été précieuse, en me prouvant qu'il était encore possible d'utiliser la forme purement classique de récit, qui autrement eût risqué peut-être de me sembler à la fois exquise et surannée, et en m'évitant de tomber dans le piège du roman proprement dit, dont la composition demande de son auteur une variété d'expérience humaine et littéraire qu'à cette époque je n'avais pas." (p. 17)

Remarquons en passant, que la stricte distinction entre le récit et le roman est une des obsessions esthétiques de Gide, et que par le même souci que Yourcenar, Gide a longtemps évité de se lancer dans la composition d'un livre proprement romanesque.

Mais ce qui importe ici c'est que dans la phrase de Yourcenar, il y a une erreur due probablement à la confusion de ses souvenirs littéraires, et une contradiction quand on la met en regard d'une des citations précédentes.

Ce que Yourcenar affirme signifie que les premiers livres de Gide étaient des récits purement classiques. Or ces livres ne sont pas des récits. Ce sont des écrits généralement courts, au langage très poétique, $d$ 'inspiration fortement symboliste, portant le plus souvent le sous-titre de traité, comme justement Le Traité $d u$ vain désir, auquel fait allusion le sous-titre d'Alexis.

D'autre part, il est contradictoire d'affirmer ne pas connaître les grands livres de Gide où le problème de l'homosexualité est ouvertement traité et reconnaître en même temps sa dette formelle par le choix purement classique du récit. Car les grands livres de Gide où le problème sexuel est, à des degrés divers, ouvertement traité se ramènent à trois: L'Immoraliste (1902); Corydon (édition définitive et courante, 1924), Les Faux-Monnayeurs(1925).

De ces trois oeuvres, seul L'Immoraliste est pur récit classique. Corydon qui analyse de manière ouverte, systématique et en termes presque scientifiques, les diverses formes de l'homosexualité, est selon la définition de Yourcenar même un essai en forme de dialogues; et Les Faux-Monnayeurs est de toute évidence un roman, seul livre que Gide lui-même consentît à classer comme tel, son "premier roman" qui resta aussi son dernier. 
Si donc Yourcenar a pu trouver un modèle formel chez Gide, ce ne peut être que L'Immoraliste qui fut justement un tournant décisif dans la production gidienne, tant sur le plan formel que sur le plan thématique. La ressemblance formelle entre Alexis et L'Immoraliste ne se limite pas d'ailleurs à la seule technique narrative, les deux récits se situent dans le cadre d'une lettre adressée à un lecteur loin du héros-narrateur.

Mais alors que signifient la parenté du titre de Yourcenar avec Corydon, et l'allusion de son sous-titre à celui de La Tentative amoureuse?

C'est que le récit de Yourcenar s'est écrit à partir de l'oeuvre de Gide, mais aussi par différenciation sinon par opposition à elle, comme il en va de toute relation intertextuelle. Et dans ces relations les différences sont en fin de compte plus significatives que les ressemblances.

Partons de l'élément commun aux deux récits qui est la lettre unique contenant toute l'aventure du héros-narrateur. L'Immoraliste enchasse la lettre dans une structure narrative qui fait de son héros Michel comme un accusé déposant devant un jury et demandant sentence qui n'est finalement pas rendue. Le récit de Gide devient ainsi une interrogation inquiète à jamais recommencée. Le héros de Yourcenar écrit une longue lettre directement adressée à sa femme dans laquelle il explique les raisons de son abandon de la vie conjugale, raisons centrées autour de son homosexualité. Il n'attend aucun jugement; au bout de son long combat, il est parvenu à la certitude qu'il agit conformément à sa nature, et qu'il a le droit et le devoir de le faire. Le récit de Yourcenar est une affirmation définitive. Peu importe dès lors que beaucoup des détails de la vie d'Alexis ressemblent à ceux de la vie de Michel, que tous deux soient élevés dans une atmosphère austère et puritaine, que tous deux descendent vers le sud à travers l'Italie après leur cérémonie de mariage, que tous deux découvrent la sensualité au contact d'enfants à la peau brune et de culture exotique, et que leurs femmes soient également soumises, vertueuses et effacées. On peut encore multiplier les similitudes. Le caractère affirmatif de l'écriture de Yourcenar pose par là même le problème de la franchise de l'expression. Le vouloir d'Alexis rencontre nécessairement un pouvoir. Peut-il dire ce qu'il veut dire et qu'il est interdit de dire? Il écrit entre autres: "On ne doit plus craindre les mots lorsqu'on a consenti aux choses. Tout simplement je ne puis pas." (p. 33) Le dilemme d'Alexis est un dilemme d'ordre langagier, le tabou qu'il doit affronter dès le moment où il prend la plume n'est pas d'ordre moral, mais d'ordre verbal. D'où les multiples détours, biais, hésitations, repentirs, comparaisons voilées, 
atermoiements, tergiversations, etc., qui constituent le tissu textuel d'Alexis. Et c'est ce dilemme qui devient le sujet propre du livre de Yourcenar. Le drame d'Alexis et de Monique ne date pas d'hier, c'est un conflit de tous les temps; ce qui change de génération c'est, autour de tels drames, "l'étendue de la zone de silence ou l'épaisseur des couches de mensonge," (p. 13) écrit Yourcenar. A l'intérieur du mariage le plus conforme aux règles sociales, "la superstition verbale" s'impose le plus tyranniquement dans les rapports sensuels entre époux. Dans L'Immoraliste, Michel ne s'adressant pas à sa femme, qui est morte d'ailleurs, il n'a pas à la ménager. La discrétion de son langage est celle du récit classique pur, et la sensualité la plus effrénée s'exprime quand il le faut avec une parfaite franchise. Les hésitations de Michel portent sur la matière morale de son récit, non sur son expression. II est perplexe devant les choses, non devant les mots. Il dit: "Je vais vous raconter ma vie, simplement, sans modestie et sans orgueil, plus simplement que si je parlais à moi-même."

C'est donc un problème de langage qui est au coeur du récit de Yourcenar, et le titre et le sous-titre y apportent d'emblée quelques éléments de solution.

La parenté de son titre avec Corydon remplit essentiellement trois fonctions: 1) Tout d'abord le lignage implicite avec l'essai de Gide est comme une réclamation de droit à traiter un sujet jusque-là tabou. La franchise presque scientifique de cette espèce d'enquête socio-culturelle qu'est Corydon sert de prolégomène à tout traitement futur de sujet, fût-ce sous forme narrative. L'existence de Corydon constitue une autorisation publique à parler de l'homosexualité.

2) En second lieu, dans l'Eglogue de Virgile, Alexis étant "l'enfant charmant"que convoite Corydon, le récit de Yourcenar annonce dès le titre que son héros va être ce qu'on pourrait appeler, faute de mieux, le pôle féminin de couples homosexuels masculins. Cette suggestion est soutenue par des notations incidentes dans le texte par Alexis lui-même:

"Il est dangereux, j'en suis sûr, pour un adolescent très sensible, d'apprendre à voir l'amour à travers des rêves de jeunes filles, même lorsqu'elles semblent pures, et qu'il s'imagine l'être aussi." (p.38)

ou ce bel exemple de la litote:

"...on ne s'éprend pas surtout de ce à quoi l'on ressemble; et ce dont je différais le plus, ce n'était pas des femmes." (p. 39)

ou encore:

"Pour tout dire, mon amie, il faudrait une audace que je me défends d'avoir; il faudrait surtout être également une femme." 
3) Enfin, tout en partant de Corydon, l'auteur autant que son personnage en rejettent le langage pseudo-scientifique, la parenté du titre n'est en ce sens qu'une rupture volontairement marquée. Yourcenar écrit dans sa préface:

"Les termes du vocabulaire scientifique, de formation récente, destinés à se démoder avec les théories qui les étayent, détériorés par une vulgarisation àoutrance qui leur enlève bientôt leurs vertus d'exactitude, ne valent que pour les ouvrages spécialisés, pour lesquels ils sont faits; ces motsétiquettes vont à l'encontre du but de la littérature qui est l'individualité dans l'expression.(p. 13)

Et Alexis souligne de son côté:

"Assurément, il suffirait pour m'expliquer de quelques termes précis, qui ne sont même pas indécents parce qu'ils sont scientifiques. Mais je ne les emploierai pas...Je sais qu'il y a des mots pour toutes les maladies, et ce dont je vous parle passe pour être une maladie. Moi-même, je l'ai cru longtemps. Mais je ne suis pas un médecin; je ne suis même plus sûr d'être un malade. La vie, Monique, est beaucoup plus complexe que toutes les définitions possibles; toute image simplifiée risque toujours d'être grossière." (p. 33)

Quant à l'allusion du sous-titre d'Alexis à celui de La Tentative amoureuse, elle signifie d'une part un choix, d'autre part un refus:

1) En premier lieu, la substitution du mot "combat" au mot "désir" signale dès le début qu'Alexis va faire le récit d'une lutte constante et finalement abanđonnée contre un "instinct", contre un déterminisme physiologique qu'il refuse longtemps de reconnaître comme tel, contre une nature fondée dans son enfance et obscurcie par son éducation. Ce combat est un choix contre le désir vague et presque inconscient qui traverse les premiers écrits de Gide, depuis Les Cahiers d'André Walter jusqu'à L'Immoraliste, y compris Le Traité du vain désir.

2) Ce choix va justement de pair avec le rejet d'un langage aussi vague que le désir des premiers personnages gidiens; et c'est le deuxième sens de l'allusion. Ce refus, Yourcenar omet de l'exprimer dans sa préface, mais il n'en est pas moins évident d'un bout à l'autre de son texte. Alexis dit, entre autres:

"Ne croyez pas non plus que j'approuve les poètes d'éviter les termes exacts, parce qu'ils ne connaissent que leurs rêves; il y a beaucoup de vrai dans les rêves des poètes, mais ils ne sont pas toute la vie." (p. 33)

Il est difficile de croire que ces trois lignes ne fassent pas intentionnellement 
écho aux premières phrases de La Tentative amoureuse:

"Nos livres n'auront pas été les récits véridiques de nousmêmes, mais plutôt nos plaintifs désirs, le souhait d'autres vies à jamais défendues, de tous les gestes impossibles. Ici j'écris un rêve qui dérangeait par trop ma pensée et réclamait une existence."

Contre le langage métaphorique des poètes et le vocabulaire pseudoscientifique, Yourcenar choisit "cette langue dépouillée, presque abstraite, à la fois circonspecte et précise, qui en France a servi durant des siècles aux prédicateurs, aux moralistes, et parfois aussi aux romanciers de l'époque classique pour traiter de ce qu'on appelait alors "les égarement des sens". (p. 14) C'est la langue même que Gide emploie dans $L$ 'Immoraliste et qu'il reconnaît comme l'héritage le plus précieux du génie classique de Bossuet, de La Bruyère ou de Madame de La Fayette. Il ne s'agit pourtant pas d'une imitation, mais le partage de cet héritage classique-toute l'oeuvre de Yourcenar en atteste la parfaite authenticité et l'originale continuité-avec un aîné qui, comme elle, en était nourri dès sa plus tendre jeunesse. Et que si Michel de L'Immoraliste ait servi de modèle à Alexis, la jeune romancière de 1929 n'en tentait pas moins une nouvelle expérimentation, après la franchise et la permission qu'octroyait Corydon. On regrette seulement qu'à l'instar de l'auteur de la trilogie de L'Ecole des femmes, Marguerite Yourcenar n'ait pas donné à sa Monique l'occasion de répondre aux confidences du mari.

1 Toutes les citations de cette oeuvre seront indiquées entre parenthèses par les numéros de pages de l'édition Gallimard, collection Folio, 1971. 